\title{
Biochemical Functions of Glutathione S-Transferase Family of Salix babylonica
}

\author{
Xiang-Lin Zhuge ${ }^{1 t}$, Hui X ${ }^{2 t}$, Zhi-Jing Xiu ${ }^{1}$ and Hai-Ling Yang ${ }^{1 *}$ \\ ${ }^{1}$ College of Biological Sciences and Technology, Beijing Forestry University, Beijing, China, ${ }^{2}$ State Key Laboratory \\ of Systematic and Evolutionary Botany, Institute of Botany, Chinese Academy of Sciences, Beijing, China
}

OPEN ACCESS

Edited by:

Toru Nakayama,

Tohoku University, Japan

Reviewed by:

Shin-ichi Ozaki,

Yamaguchi University, Japan

Satoshi Yamashita,

Kanazawa University, Japan

*Correspondence:

Hai-Ling Yang

yhailing77@163.com

${ }^{t}$ These authors have contributed

equally to this work

Specialty section:

This article was submitted to

Plant Metabolism

and Chemodiversity,

a section of the journal

Frontiers in Plant Science

Received: 09 June 2019

Accepted: 13 March 2020

Published: 03 April 2020

Citation:

Zhuge $X-L, X u H$, Xiu Z-J and Yang H-L (2020) Biochemical

Functions of Glutathione

S-Transferase Family of Salix

babylonica. Front. Plant Sci. 11:364.

doi: 10.3389/fp/s.2020.00364
Glutathione S-transferases (GSTs) are ubiquitous enzymes that are encoded by a large gene family, and they contribute to the detoxification of endogenous or xenobiotic compounds and oxidative stress metabolism in plants. Although the GSTs gene family has been reported in many land plants, our knowledge of the evolution and function of the willow GSTs is still limited. In this study, 22 full-length GST genes were cloned from Salix babylonica and divided into three classes based on the conserved domain analysis, phylogenetic tree and gene structure: tau, phi and DHAR. The tissue-specific expression patterns were substantially different among the tau and phi GSTs. The Salix GST proteins showed functional divergences in the substrate specificities, substrate activities and kinetic characteristics. The site-directed mutagenesis studies revealed that a single amino acid mutation (lle/Val53 $\rightarrow$ Thr53) resulted in the lowest activity of SbGSTU7 among the Salix GSTs. These results suggest that non-synonymous substitution of an amino acid at the putative glutathione-binding site may play an important role in the divergence of enzymatic functions of Salix GST family.

Keywords: glutathione S-transferase, gene expression, enzyme activity, kinetic analysis, salix babylonica

\section{INTRODUCTION}

Glutathione S-transferases (GSTs; EC 2.5.1.18) are encoded by a large gene family and widely distributed in both prokaryotes and eukaryotes. They are multifunction proteins whose functions include detoxification, since they mainly catalyze the detoxification of a series of xenobiotics by conjugating the reduced glutathione (GSH) to various hydrophobic and electrophilic compounds (Dirr et al., 1994; Armstrong, 1997). GSTs are divided into at least four major families of proteins: cytosolic GSTs, mitochondrial GSTs, microsomal GSTs and bacterial fosfomycin-resistance proteins (Oakley, 2005; Allocati et al., 2009), with the cytosolic GST family being the most abundant of the four groups in plants. Based on the amino acid sequence similarity, gene structure and substrate specificity, the plant GSTs have been divided into eight classes, including phi, tau, theta, zeta, lambda, dehydroascorbate reductase (DHAR), tetrachlorohydroquinone dehalogenase (TCHQD) and $\gamma$-subunit of the eukaryotic translation elongation factor 1B (EF1Bg) (Edwards et al., 2000; Oakley, 2005; Lan et al., 2009). In addition, two new classes (hemerythrin GST and iota GST) were recently identified in nonvascular plants (Liu et al., 2013). The GSTs classes of tau and phi are the most abundant in terrestrial plants among these ten GST classes, except in non-vascular plants that have no tau GSTs (Frova, 2006; Liu et al., 2013; Labrou et al., 2015); they were observed only in plants (Basantani and Srivastava, 2007) and are largely responsible for the detoxification processes 
of endogenous or xenobiotic compounds and oxidative stress metabolism, they also help to select herbicide in crops and weeds (Skipsey et al., 2005; Benekos et al., 2010; Cummins et al., 2011, 2013). In addition to detoxification, GSTs are also capable of regulating the redox homeostasis in cells to protect them against UV radiation and oxidative stress (Loyall et al., 2000; Roxas et al., 2000; Jiang et al., 2010). The other typical functions of GSTs include the involvement in the process of cell signaling and cellular apoptosis and the contribution to the biosynthesis and transport of secondary metabolites (Loyall et al., 2000; Dixon et al., 2010).

The genus Salix, a member of the Salicaceae family, is widespread throughout China, and represents an essential part of the urban and rural ecosystems (Wu et al., 2015). The species of Salix are highly adaptable ones that can grow in various ecological environments (mountains, plains, sand dunes and polar regions), and could be considered as excellent species for the phytoremediation of heavy metals pollution (Yildirim and Kasim, 2016). GSTs are considered to crucially contribute to the stress tolerance and heavy metals resistance, and although this gene family has been reported in many land plants, our knowledge concerning the evolution and function of willow GSTs is limited. With the aim to further understand the GST supergene family in weeping willow (Salix babylonica), we studied its structural and functional characterizations in this study. S. babylonica is considered to be a promising species for bioenergy production due to the high biomass yields through the short-rotation coppice systems (Brereton et al., 2012; Cunniff et al., 2015). It is widely planted for the construction of shelterbelts, which protect the agricultural land in the oasis of the Gobi Desert (Dickmann and Kuzovkina, 2014). Therefore, it is an important genus from the economic and ecological aspects. In this study, 22 full-length GST genes from S. babylonica were cloned and divided into the classes of tau, phi and DHAR. The gene structural features, tissue-specific expression patterns, enzymatic characteristics and site-directed mutagenesis assay of willow GSTs were integrated in this study to provide a comprehensive understanding of the S. babylonica GST gene family.

\section{MATERIALS AND METHODS}

\section{GST Genes Identification and Nomenclature}

In order to identify the GST genes from $S$. babylonica, the transcriptome database (unpublished) of $S$. babylonica was searched using the TBLASTN program with the default algorithm parameters and 81 full-length GST protein sequences of Populus trichocarpa. The GST candidates of S. babylonica were then looked up in the National Center for Biotechnology Information (NCBI) Conserved Domains Database ${ }^{1}$ to confirm the presence of typical GST N- and C-terminal domains in the protein structures. Next, primers were designed, based on the identified GST gene sequences, to amplify the genomic and

${ }^{1}$ http://www.ncbi.nlm.nih.gov/Structure/cdd/wrpsb.cgi coding sequences of each S. babylonica GST (Supplementary Table S1), such that the GST genomic sequences and coding sequences were amplified from the S. babylonica DNA and CDNA, respectively. The amplified sequences were then cloned into a pEASY-T3 vector (TransGen, Beijing, China), and sequenced in both directions. Finally, the amplified coding sequences of the S. babylonica GST genes were mapped to their corresponding genomic sequences to verify the intron/exon structures.

The S. babylonica GSTs were named according to the system described by Edwards et al. (2000), such that the name of each gene consists of three parts: the source of the organism, the subfamily name and a progressive number. In this study, we used $S b$ to represent Salix babylonica, then the subfamily name was denoted by GST plus the logogram of each class. For example, GSTU, GSTF, DHAR, correspond to the tau, phi, DHAR classes, respectively, and the full phi GST genes names are SbGSTF1, SbGSTF2, etc.

\section{Phylogenetic Analysis and Homology Modeling}

The GST protein sequences were separated into two distinct parts according to the N-terminal and C-terminal domains. The protein sequences of the full-length, $\mathrm{N}$-terminal domain and the C-terminal domain were, respectively, aligned using the MUSCLE online service. Next, the alignments were manually further adjusted using the BioEdit v7.0.0 software (Alzohairy, 2011), then the pairwise alignments for the sequence identities were analyzed using the Sequence Identity Matrix in the BioEdit software. The Jones, Taylor, and Thornton (JTT) amino acid substitution model was selected by the ModelGenerator program version 0.85 . The phylogenetic tree was constructed following a maximum-likelihood procedure using the PhyML software version 3.1 (Guindon et al., 2009). There were 100 bootstrap replicates. The GRX2 protein was used as an outgroup (Oakley, 2005). The structure of the SbGSTU7 gene was built using the GmGSTU10-10 (Protein Data Bank accession number 4CHS) as a template by the SWISS-MODEL software ${ }^{2}$. The simulated structure of the SbGSTU7 gene was then manually processed using the Discovery Studio 4.0 Client software.

\section{Tissue-Specific Expression Patterns of the GST Genes}

To extract the total RNA, we sampled the primary leaves, mature leaves, phloem, roots, buds and flowers from three hydroponic S. babylonica trees. The primary leaves with a length of 2$3 \mathrm{~cm}$ that were newly-expanded and the mature leaves with a length of 10-12 cm were collected from the shoot top and the middle of the shoot, respectively. After the inverse transcription of the RNA using the RNA PCR Kit (AMV) (TaKaRa, Dalian, China), the real-time PCR (qRT-PCR) was performed using the SYBR Green PCR master mix (Tiangen) and Bio-Rad iQ5 Real-Time PCR system (Bio-Rad, United States). Three biological replicates and three technical ones were performed for each qRT-PCR procedure. The S. babylonica actin gene

\footnotetext{
${ }^{2}$ https://swissmodel.expasy.org/interactive
} 
(Supplementary Table S2) was used as the internal reference. The relative expression levels were calculated using the $2^{-\Delta \mathrm{Ct}}$ method (Livak and Schmittgen, 2001), where $\Delta$ CT denotes the difference between the target and housekeeping gene actin in the threshold cycles: $\Delta \mathrm{CT}=\mathrm{CT}$ (a target gene) - CT (actin gene). The specific qRT-PCR primers for the S. babylonica GSTs are listed in Supplementary Table S2.

\section{Expression and Purification of the GST Proteins}

In this study, 22 GSTs (14 tau, 6 phi, and 2 DHAR) were selected for the enzymatic activity assay. Each of these 22 GST proteins was subcloned into a pET30a expression vector to obtain a $6 \times$ His-tag. The primers that were used to construct the GST expression vectors are listed in Supplementary Table S3. After confirming the sequence of the recombinant vectors, the vectors were introduced into Escherichia coli BL21 (DE3) cells. The BL21 cells, containing the recombinant vectors, were then cultured to an optical density $\left(\mathrm{A}_{600}\right)$ of 0.5 , and isopropyl- $\beta-\mathrm{D}$ thiogalactopyranoside (IPTG) was added to the culture to induce the expression of the GST proteins. The final concentration of IPTG was $0.1 \mathrm{mM}$. After the induction $\left(12 \mathrm{~h}\right.$ at $\left.20^{\circ} \mathrm{C}\right)$, the cells were harvested by centrifugation $\left(10,000 \times \mathrm{g}, 3 \mathrm{~min}, 4^{\circ} \mathrm{C}\right)$ and resuspended in binding buffer $(20 \mathrm{mM}$ sodium phosphate, $0.5 \mathrm{M}$ $\mathrm{NaCl}$ and $20 \mathrm{mM}$ imidazole, $\mathrm{pH}$ 7.4). The cells were disrupted by sonication in ice-cold binding buffer. Next, the homogenate was centrifuged $\left(10,000 \times g, 10 \mathrm{~min}, 4^{\circ} \mathrm{C}\right)$. In order to check the solubility of the recombinant proteins, the resulting particulate material and a small portion of the supernatant were analyzed using SDS-PAGE. Regarding the soluble recombinant proteins, the rest of the supernatant was loaded onto a Nickel-Sepharose High Performance column (GE Healthcare BioSciences), and the GST proteins were then eluted with elution buffer $(20 \mathrm{mM}$ sodium phosphate, $0.5 \mathrm{M} \mathrm{NaCl}$ and $500 \mathrm{mM}$ imidazole, $\mathrm{pH} 7.4$ ).

In order to obtain the mutated proteins, the site-directed mutagenesis of the protein sequences was performed by the methods that were previously reported (Zeng and Wang, 2005). The primers that were used to construct the mutants are shown in Supplementary Table S3.

\section{Activity and Kinetics Assays of the S. babylonica GST Proteins}

The enzymatic activity assay of the purified S. babylonica GST proteins were performed using a 752 UV visible single beam spectrophotometer (Jinghua, Shanghai, China). According to the methods described by Habig et al. (1974) and Ricci et al. (1994), we were able to determine the activity of four conventional substrates: 1-chloro-2,4-dinitrobenzene (CDNB), 7-chloro-4-nitrobenzo-2-oxa-1,3-diazole (NBD-Cl), 1,2dichloro-4-nitrobenzene (DCNB) and 4-nitrobenzyl chloride (NBC). The cumene hydroperoxide (Cum-OOH) was selected to determine the GSH-dependent peroxidase activities, the dehydroascorbic acid (DHA) was selected to measure the dehydroascorbate reductase activities, and the diphenyl ether (fluorodifen) was used as a substrate to determine the herbicide detoxification activities. These activities were determined following the method of Edwards and Dixon (2005), and the reactions were performed in the following buffers: $50 \mathrm{mM}$ potassium phosphate buffer $\mathrm{pH} 6.5$ for CDNB and NBC, $50 \mathrm{mM}$ potassium phosphate buffer $\mathrm{pH} 7.5$ for DCNB, $100 \mathrm{mM}$ sodium acetate buffer $\mathrm{pH} 5.0$ for NBD-Cl, $100 \mathrm{mM}$ potassium phosphate buffer $\mathrm{pH} 6.5$ for DHA, $50 \mathrm{mM}$ glycine $\mathrm{pH} 9.5$ for fluorofen, $50 \mathrm{mM}$ potassium phosphate buffer $\mathrm{pH} 7.0$ containing $1 \mathrm{mM}$ ethylenediamine tetraacetic acid and $1 \mathrm{mM}$ sodium azide for Cum-OOH. The protein concentrations were determined by absorption at $280 \mathrm{~nm}$.

In order to examine the steady-state kinetic parameters of the Salix tau GSTs, the apparent $K_{\mathrm{m}}$ values for GSH and CDNB were separately determined. The concentration of GSH ranged from $0.1 \mathrm{mM}$ to $1 \mathrm{mM}$, and the concentration of CDNB was fixed at $1.0 \mathrm{mM}$ to determine the $K_{\mathrm{m}}^{\mathrm{GSH}}$. To determine the $K_{\mathrm{m}}^{\mathrm{CDNB}}$ value, the concentration of CDNB ranged from $0.1 \mathrm{mM}$ to $2.0 \mathrm{mM}$, and the concentration of GSH was fixed at $1.0 \mathrm{mM}$. The kinetic parameters were calculated using nonlinear regression analysis by the Hyper32 program available online at http://hyper32. software.informer.com/. All the activity and kinetics assays were determined at $25{ }^{\circ} \mathrm{C}$ and performed at least three times. The statistical analysis for the enzyme activities between the wild type and mutant proteins were analyzed using the SPSS software ver. 16.0 (IBM Corporation, Armonk, NY, United States).

\section{RESULTS}

\section{Identification of the Salix babylonica GST Genes}

Based on the analysis by the conserved domain of the National Center for Biotechnology Information (NCBI), 22 putative Salix GST proteins were identified to belong to the GST classes of tau, phi or DHAR, and their coding sequences were successfully cloned from Salix babylonica (Table 1). The phylogenetic relationships revealed that the 22 Salix GSTs were divided into three distinct clades with high bootstrap supports (Figure 1A). In order to further confirm the subfamily designations of these GSTs, the phylogenetic tree was constructed using 22 Salix and 81 Populus GSTs, which indicates that the tau, phi and DHAR GSTs of Salix were clustered with the corresponding classes of Populus GSTs with high bootstrap support (Figure 2). The results of the conserved domain analysis and the phylogenetic trees showed that among the 22 Salix GSTs, 14, 6, and 2 members belonged to the classes of tau, phi and DHAR, respectively.

\section{Sequence and Structural Characteristics of Salix GSTs}

This study identified the gene structures of 20 Salix GSTs (Figure 1B), while the gene structure identification of the remaining 2 GSTs, SbGSTF2, and SbDHAR1, failed due to unsuccessful cloning of their genomic sequences. The gene structures were conserved among the Salix GSTs from the tau and phi classes, respectively (Figure 1B). All the 14 tau GST genes contained two exons, and all the six phi GST genes contained three exons. The DHAR GSTs contained more introns than 
TABLE 1 | The GST genes cloned from Salix babylonica.

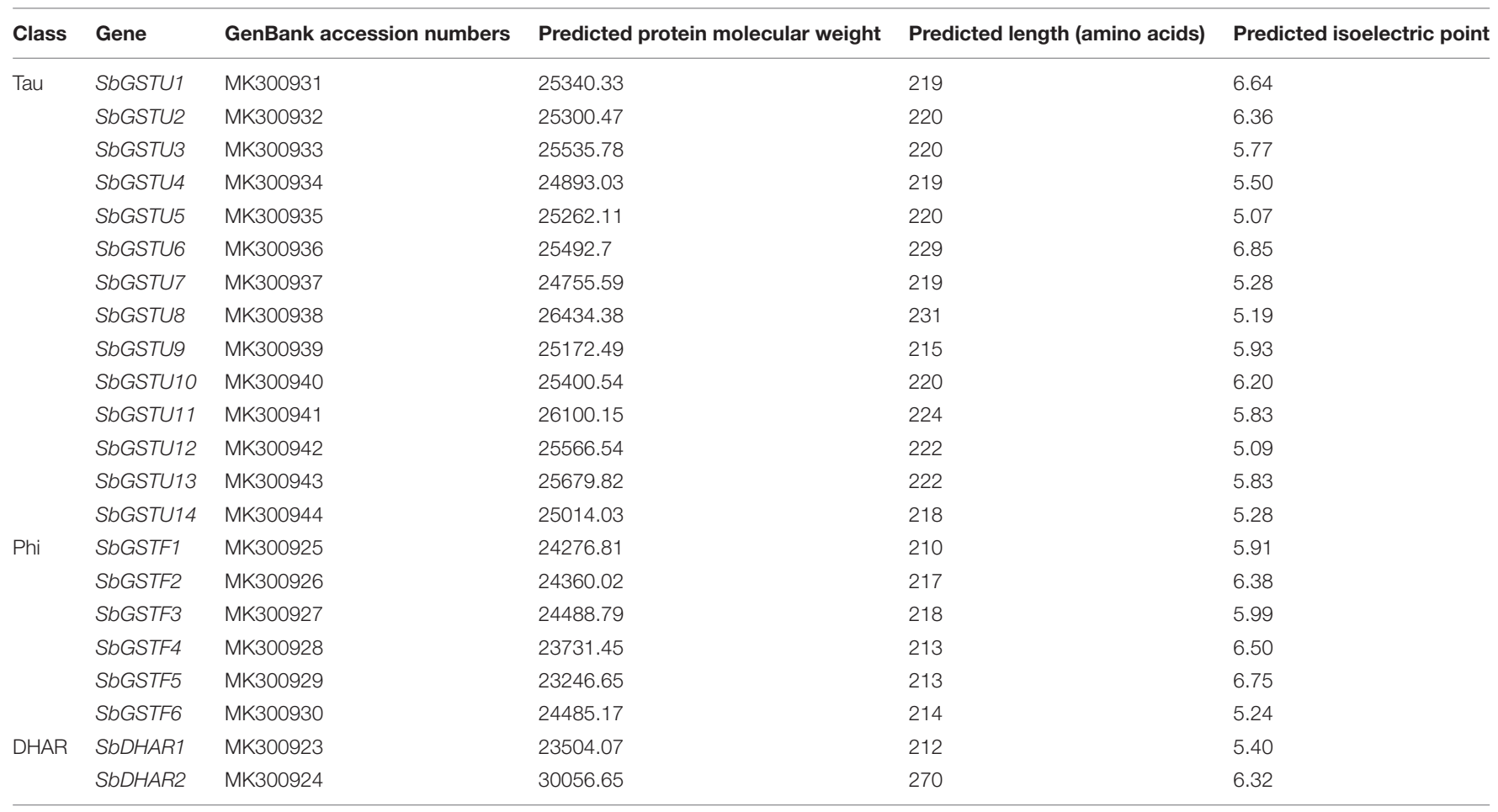

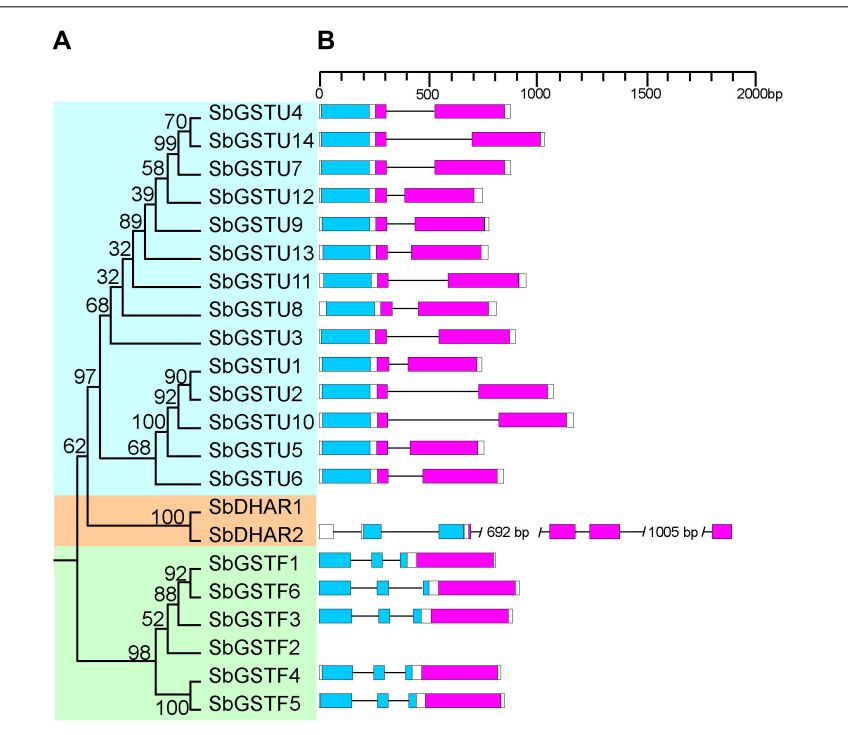

FIGURE 1 | Phylogenetic tree of Salix GSTs (A) and their gene structures (B). The tree was reconstructed with protein sequences using $\mathrm{ML}$ procedure with JTT model. Numbers at each node in the ML tree signify bootstrap values. Different GST classes are shaded with different colors. The GST N-terminal domain and C-terminal domain are highlighted in blue and purple, respectively. Introns are indicated as lines.

the tau and phi GSTs. The gene of SbDHAR2 contained five introns which was similar to the poplar DHAR class GSTs. The obtained gene structures in different classes further supported the class designations.
The 22 Salix GST proteins' lengths ranged from 210 to 270 amino acids, and their deduced protein molecular weights were between 23.2 and $30.1 \mathrm{kDa}$ (Table 1). The GST proteins were divided into two distinct domains: The N-terminal domain and the C-terminal domain. The sequence of 14 tau GSTs proteins showed $31.2-75.0 \%$ pairwise sequence identities, such that the pairwise protein sequence identity of the $\mathrm{N}$-terminal and C-terminal domains was $43.2-88.4 \%$ and $21.0-71.6 \%$, respectively. On the other hand, the pairwise sequence identity of 6 phi GST proteins was $37.3-62.1 \%$, such that it was $41.2-$ $64.1 \%$ for their N-terminal domain protein sequences and 36.0$64.2 \%$ for the C-terminal domain. Compared with the C-terminal domain sequences, the $\mathrm{N}$-terminal domain sequences were much more conserved for the Salix tau and phi GSTs (Figure 3).

\section{Expression Patterns of Salix GSTs}

We examined the expression patterns of 22 Salix GSTs under normal growth conditions using the quantitative real-time PCR method (Figure 4). The expressions pattern of the Salix tau and phi GSTs were different (MRPP test $P<0.06$ ), since they were more variable among the Salix tau GSTs compared with the phi GSTs. The Salix tau GSTs were divided into two clades (Figure 1A). Interestingly, all the nine GST genes in clade I, including the genes of SbGSTU7 and SbGSTU12, showed a much higher expression level in the root tissues than that in the other examined tissues (Figure 4). For the genes in clade II, the genes of SbGSTU1 and SbGSTU2 showed a higher expression level in the primary leaf tissues than that in the other examined tissues. The gene of SbGSTU5 showed a specific high expression level in the root tissues, while the gene of SbGSTU6 was highly expressed in 


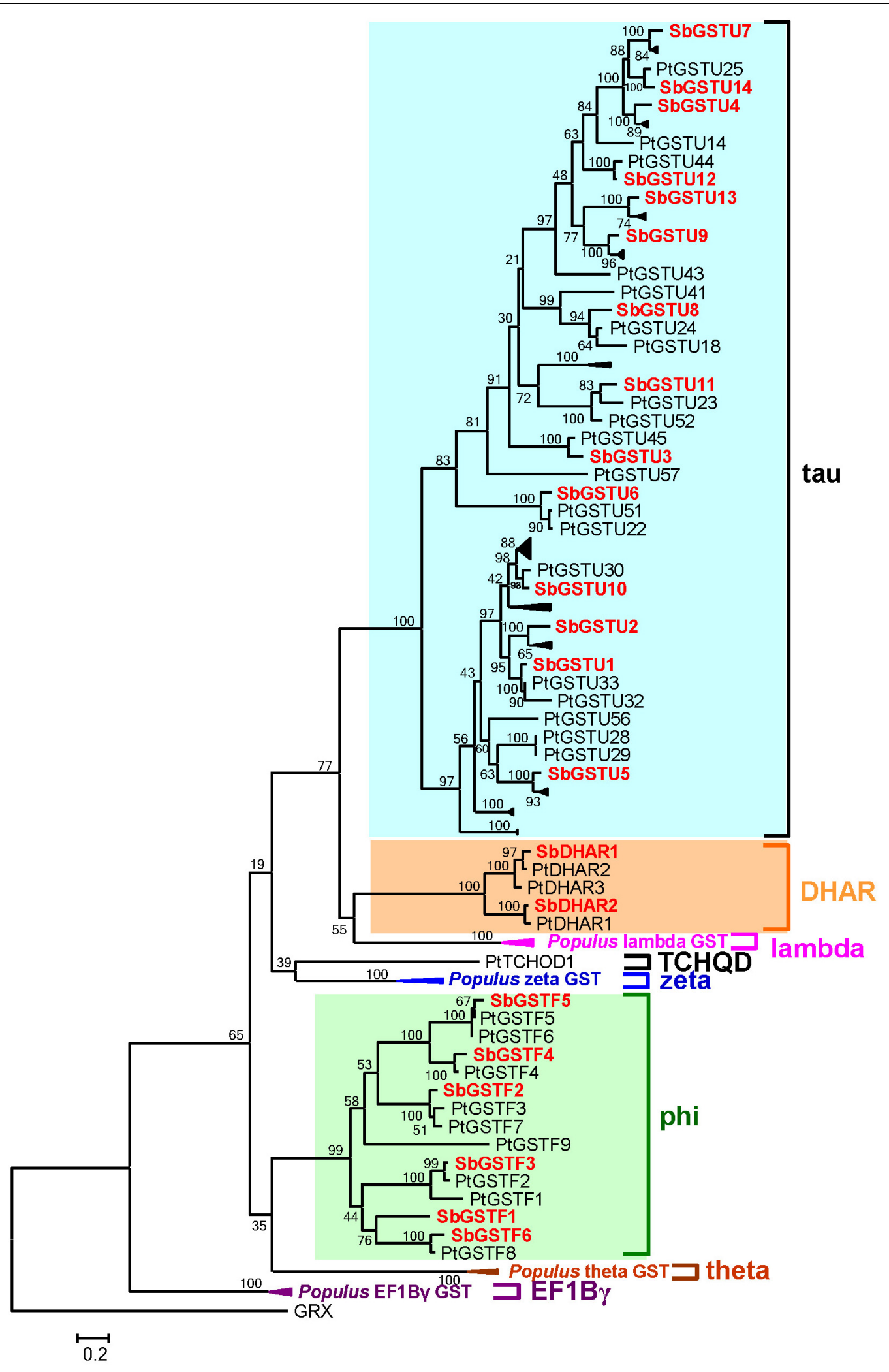

FIGURE 2 | Phylogenetic relationships between Salix and Populus GSTs. The tree was reconstructed with protein sequences using ML procedure with JTT model. Salix GSTs are represented by red letters. 

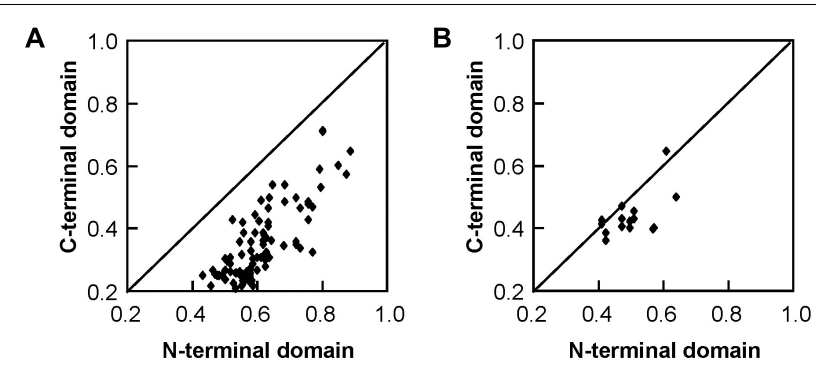

FIGURE 3 | Pairwise protein sequence identity plots for the C-terminal domain versus the N-terminal domain of Salix tau (A) and phi (B) GSTs. Each dot represents the pairwise protein sequence identity for $\mathrm{C}$-terminal domain versus the $\mathrm{N}$-terminal domain between two GST proteins from the same class.

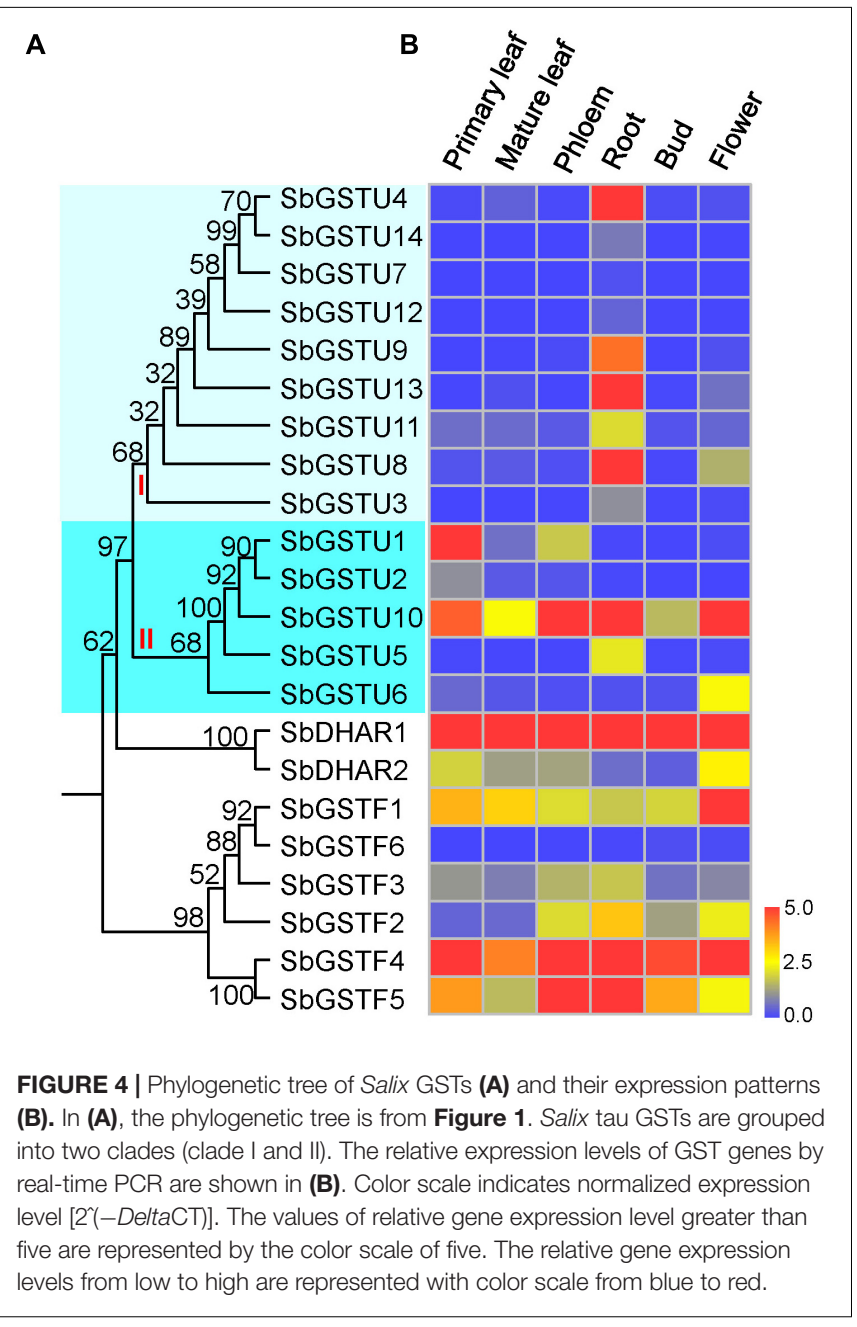

the flower tissues. The gene of SbGSTU10 was highly expressed in all the examined tissues (Figure 4).

Regarding the Salix phi GSTs, the gene of SbGSTF1 showed a high expression level in the flower tissues (Figure 4), and the SbGSTF5 and SbGSTF3 genes were highly expressed in the phloem and root tissues than the other examined tissues. Among all the six Salix phi GSTs, only the SbGSTF6 gene showed a low expression level in all the examined tissues. Similar to SbGSTF4, the gene of SbDHAR1 was highly expressed in all the tissues. Finally, $S b D H A R 2$ showed a high expression level in the primary leaf and flower tissues.

\section{Enzyme Activities of the Salix GST Proteins}

In order to investigate the substrate specificities and activities of the Salix GST proteins, all the 22 Salix GST proteins were selected in this study for protein expression and purification. Eighteen Salix GSTs (all the 14 tau GSTs, 3 phi GSTs and the SbDHAR2 gene) were expressed as soluble proteins in Escherichia coli (E. coli), while 3 phi GSTs (The genes of SbGSTF1, 3, and 6 ) and the SbDHAR1 gene were expressed as inclusion bodies in E. coli. In order to assay the enzymatic activities of Salix GSTs, seven GST substrates were used: CDNB, NBD-Cl, DCNB, NBC, fluorodifen, Cum-OOH, and DHA (Figure 5).

All the 14 Salix tau GSTs showed activities to the two substrates of CDNB and NBD-Cl. Among these GSTs, SbGSTU1 showed the widest substrate spectrum with enzymatic activities to five substrates, and SbGSTU6 showed the highest enzymatic activities to the CDNB, NBD-Cl and fluorodifen substrates, to which SbGSTU7 displayed the lowest enzymatic activities. Only SbGSTU13 showed an activity toward the substrate $\mathrm{Cum}-\mathrm{OOH}$, and only SbGSTU1 and SbGSTU10 displayed activities toward the substrate DCNB.

All the 3 purified Salix phi GSTs showed activities toward the CDNB and NBD-Cl substrates and had no activity toward the Cum-OOH and DHA substrates. SbGSTF4 and SbGSTF5 displayed activities to the DCNB substrate, while SbGSTF2 did not. On the other hand, SbGSTF2 and SbGSTF5 showed activities toward NBC, while SbGSTF4 did not. Only SbGSTF2 exhibited an enzymatic activity toward the fluorodifen substrate.

\section{Kinetic Properties of the Salix GSTs}

The $K_{\mathrm{m}}$ values indicate the affinity of the enzyme and its substrates, and GSTs can catalyze the conjugation of reduced GSH to the electrophilic group of a wide range of hydrophobic compounds. In this study, the steady-state kinetic parameters of 14 purified Salix tau GSTs were determined (Table 2). Except for SbGSTU2 and SbGSTU7, the apparent $K_{\mathrm{m}}^{\mathrm{GSH}}$ values of the Salix tau GSTs ranged from 0.140 to $0.893 \mathrm{mM}$, while the range of variations of the apparent $K_{\mathrm{m}}^{\mathrm{CDNB}}$ values was from 0.156 to $5.121 \mathrm{mM}$, which was greater than the variations of the $K_{\mathrm{m}}^{\mathrm{GSH}}$ values; this suggests that the tau GSTs had similar, high affinities for the GSH substrate than the hydrophobic substrates. Among the 14 Salix tau GSTs, SbGSTU6 showed the highest $k_{\text {cat }}^{\mathrm{CDNB}}$ value, SbGSTU10 had the highest $\left(k_{\mathrm{cat}} / K_{\mathrm{m}}\right)^{\mathrm{CDNB}}$ value, and SbGSTU7 had the lowest $k_{\text {cat }}^{\mathrm{CDNB}}$ and $\left(k_{\mathrm{cat}} / K_{\mathrm{m}}\right)^{\mathrm{CDNB}}$ values.

\section{Site-Directed Mutagenesis of the SbGSTU6 and SbGSTU7 Proteins}

Among the 14 Salix tau GSTs, SbGSTU7 showed the lowest enzymatic activities to the CDNB, NBD-Cl and fluorodifen substrates, to which SbGSTU6 had the highest enzymatic activities (Figure 5). The predicted tertiary structure of SbGSTU7 


\begin{tabular}{|c|c|c|c|c|c|c|c|c|}
\hline \multirow{2}{*}{\multicolumn{2}{|c|}{ GSTs }} & \multicolumn{3}{|c|}{ Specific activities ( $\mu \mathrm{mol} \mathrm{min}^{-1} \mathrm{mg}^{-1}$ ) } & \multirow[b]{2}{*}{ NBC } & \multirow[b]{2}{*}{ Fluorodifen } & \multirow[b]{2}{*}{ Cum-OOH } & \multirow[b]{2}{*}{ DHA } \\
\hline & & CDNB & NBD-Cl & DCNB & & & & \\
\hline & SbGSTU4 & $4.47 \pm 0.17$ & $1.64 \pm 0.02$ & n.d. & $0.55 \pm 0.08$ & $1.81 \pm 0.01$ & n.d. & n.d. \\
\hline & SbGSTU14 & $10.03 \pm 0.19$ & $3.18 \pm 0.10$ & n.d. & n.d. & n.d. & n.d. & n.d. \\
\hline & SbGSTU7 & $0.11 \pm 0.01$ & $0.12 \pm 0.01$ & n.d. & n.d. & $0.20 \pm 0.01$ & n.d. & n.d. \\
\hline & SbGSTU12 & $0.54 \pm 0.01$ & $2.60 \pm 0.01$ & n.d. & n.d. & $0.92 \pm 0.02$ & n.d. & n.d. \\
\hline & SbGSTU9 & $4.9 \pm 0.09$ & $7.26 \pm 0.34$ & n.d. & n.d. & $1.84 \pm 0.01$ & n.d. & n.d. \\
\hline & SbGSTU13 & $0.38 \pm 0.01$ & $2.85 \pm 0.06$ & n.d. & n.d. & $2.93 \pm 0.01$ & $0.12 \pm 0.01$ & n.d. \\
\hline & SbGSTU11 & $3.94 \pm 0.05$ & $0.40 \pm 0.004$ & n.d. & n.d. & n.d. & n.d. & n.d. \\
\hline & SbGSTU8 & $0.25 \pm 0.01$ & $0.85 \pm 0.01$ & n.d. & n.d. & $0.79 \pm 0.01$ & n.d. & n.d. \\
\hline & SbGSTU3 & $11.14 \pm 0.29$ & $3.02 \pm 0.02$ & n.d. & $0.84 \pm 0.02$ & $8.62 \pm 0.01$ & n.d. & n.d. \\
\hline & SbGSTU1 & $0.97 \pm 0.02$ & $0.92 \pm 0.01$ & $0.27 \pm 0.01$ & $0.65 \pm 0.05$ & $0.72 \pm 0.02$ & n.d. & n.d. \\
\hline & SbGSTU2 & $0.69 \pm 0.01$ & $1.44 \pm 0.03$ & n.d. & n.d. & $0.61 \pm 0.01$ & n.d. & n.d. \\
\hline & SbGSTU10 & $8.16 \pm 0.10$ & $7.94 \pm 0.03$ & $0.26 \pm 0.01$ & n.d. & $17.18 \pm 0.04$ & n.d. & n.d. \\
\hline & SbGSTU5 & $1.65 \pm 0.01$ & $2.41 \pm 0.02$ & n.d. & $0.55 \pm 0.02$ & $11.88 \pm 0.14$ & n.d. & n.d. \\
\hline & SbGSTU6 & $19.58 \pm 0.08$ & $15.69 \pm 0.15$ & n.d. & n.d. & $32.95 \pm 0.61$ & n.d. & n.d. \\
\hline & SbDHAR1 & n.a. & n.a. & n.a. & n.a. & n.a. & n.a. & n.a. \\
\hline & SbDHAR2 & n.d. & n.d. & n.d. & n.d. & n.d. & n.d. & $1.42 \pm 0.01$ \\
\hline & SbGSTF1 & n.a. & n.a. & n.a. & n.a. & n.a. & n.a. & n.a. \\
\hline & SbGSTF6 & n.a. & n.a. & n.a. & n.a. & n.a. & n.a. & n.a. \\
\hline & SbGSTF3 & n.a. & n.a. & n.a. & n.a. & n.a. & n.a. & n.a. \\
\hline & SbGSTF2 & $2.82 \pm 0.07$ & $3.14 \pm 0.04$ & n.d. & $0.66 \pm 0.08$ & $0.33 \pm 0.01$ & n.d. & n.d. \\
\hline & SbGSTF4 & $2.83 \pm 0.05$ & $4.02 \pm 0.08$ & $0.11 \pm 0.01$ & n.d. & n.d. & n.d. & n.d. \\
\hline & SbGSTF5 & $0.41 \pm 0.01$ & $3.20 \pm 0.06$ & $0.11 \pm 0.01$ & $0.91 \pm 0.18$ & n.d. & n.d. & n.d. \\
\hline
\end{tabular}

FIGURE 5 | Enzymatic activities of Salix GSTs. The phylogenetic tree is from Figure 1. Values of enzymatic activities to different substrates are means \pm SD as calculated from three replicates. n.d., no activity detected; n.a., not assayed.

TABLE 2 | Steady-state kinetic parameters of Salix tau GSTs.

\begin{tabular}{|c|c|c|c|c|c|c|}
\hline GSTs & $\begin{array}{c}K_{\mathrm{m}}^{\mathrm{GSH}} \\
\mathrm{mM}\end{array}$ & $\begin{array}{c}k_{\mathrm{cat}}^{\mathrm{GSH}} \\
\mathrm{s}^{-1}\end{array}$ & $\begin{array}{c}\left(k_{\text {cat }} / K_{\mathrm{m}}\right)^{\mathrm{GSH}} \\
\mathrm{mM}^{-1} \mathrm{~s}^{-1}\end{array}$ & $\begin{array}{c}K_{\mathrm{m}}^{\mathrm{CDNB}} \\
\mathrm{mM}\end{array}$ & $\begin{array}{c}k_{\mathrm{cat}}^{\mathrm{CDNB}} \\
\mathrm{s}^{-1}\end{array}$ & $\begin{array}{c}\left(k_{\text {cat }} / K_{\mathrm{m}}\right)^{\mathrm{CDNB}} \\
\mathrm{mM}^{-1} \mathrm{~s}^{-1}\end{array}$ \\
\hline SbGSTU1 & $0.473 \pm 0.023$ & $6.283 \pm 0.149$ & 13.277 & $0.201 \pm 0.004$ & $6.056 \pm 0.081$ & 30.102 \\
\hline SbGSTU3 & $0.140 \pm 0.004$ & $16.714 \pm 0.127$ & 119.300 & $1.003 \pm 0.033$ & $28.819 \pm 0.783$ & 28.733 \\
\hline SbGSTU4 & $0.146 \pm 0.002$ & $2.963 \pm 0.025$ & 20.296 & $5.121 \pm 0.106$ & $24.482 \pm 0.371$ & 4.781 \\
\hline SbGSTU5 & $0.240 \pm 0.011$ & $3.376 \pm 0.011$ & 14.073 & $2.364 \pm 0.080$ & $7.782 \pm 0.071$ & 3.292 \\
\hline SbGSTU7 & $1.183 \pm 0.059$ & $0.804 \pm 0.025$ & 0.679 & $2.074 \pm 0.165$ & $1.415 \pm 0.015$ & 0.682 \\
\hline SbGSTU8 & $0.893 \pm 0.026$ & $1.197 \pm 0.021$ & 1.340 & $2.628 \pm 0.669$ & $2.061 \pm 0.148$ & 0.784 \\
\hline SbGSTU9 & $0.208 \pm 0.006$ & $7.564 \pm 0.089$ & 36.365 & $2.785 \pm 0.060$ & $21.060 \pm 0.391$ & 7.562 \\
\hline SbGSTU10 & $0.284 \pm 0.009$ & $7.391 \pm 0.028$ & 26.053 & $0.156 \pm 0.002$ & $16.183 \pm 0.178$ & 104.004 \\
\hline SbGSTU11 & $0.732 \pm 0.082$ & $16.302 \pm 0.326$ & 22.285 & $3.372 \pm 0.538$ & $22.472 \pm 2.083$ & 6.664 \\
\hline SbGSTU12 & $0.333 \pm 0.054$ & $1.314 \pm 0.013$ & 3.947 & $2.144 \pm 0.272$ & $1.934 \pm 0.043$ & 0.902 \\
\hline
\end{tabular}

Values shown are means $\pm S D$, calculated from three replicates. 

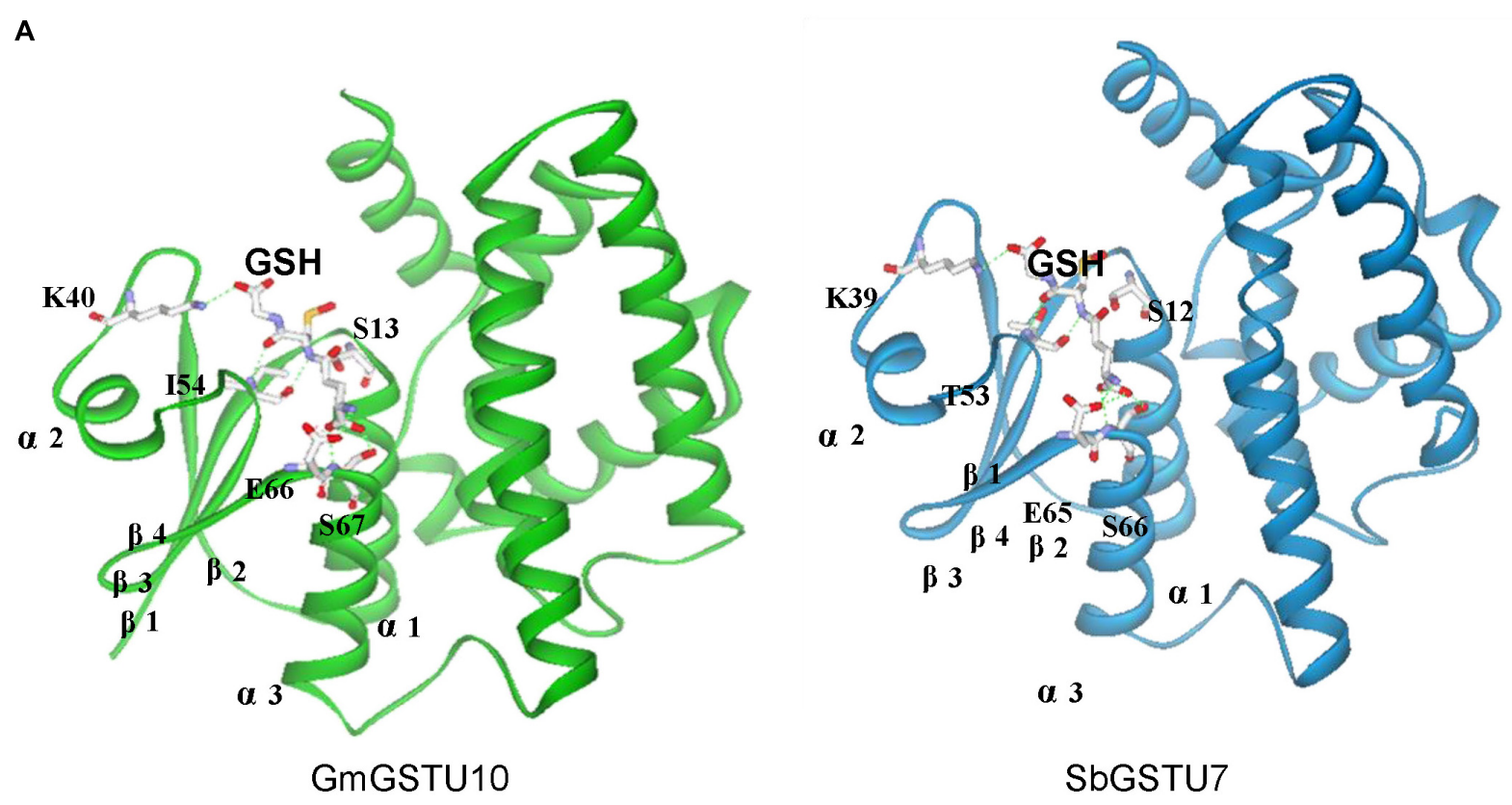

SbGSTU7

B
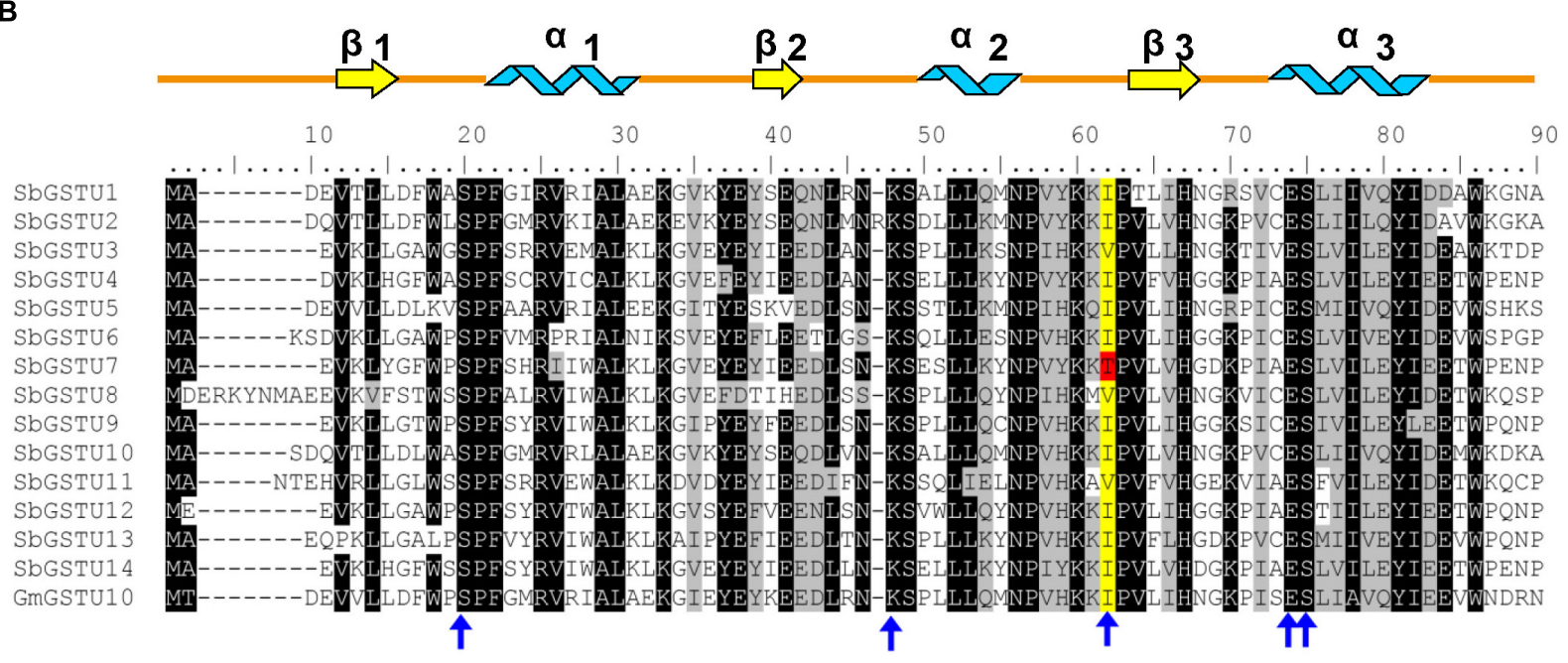

FIGURE 6 | Structural comparison of G-site residues (A) and N-terminal amino acid sequence alignment of the Salix tau GSTs (B). The structure of GmGSTU10 (template, PDB No. 4CHS) and SbGSTU7 (stimulated) are presented in (A). The amino acid structures of GSH-binding sites are shown with sticks. In (B), the conserved amino acids are shaded with black and gray. The G-sites of SbGSTU7 are marked with blue arrows. One conserved residue of G-sites (Ile/Nal, alignment position 62) is shaded with yellow and the Thr substitution in SbGSTU7 of this residue is shaded with red. Alpha helices and beta strands are represented as blue ribbons and yellow arrows, respectively.

was similar to that of the Glycine max tau GST (GmGSTU10, Protein Data Bank code (ID) No.: 4CHS). The GSH-binding sites of GmGSTU10 were Ser13, Lys40, Ile54, Glu66, and Ser67 (Figure 6A). Based on the $\mathrm{N}$-terminal amino acid sequence identity, these sites were highly conserved among the 14 Salix tau GSTs (Figure 6B). However, the conserved Ile/Val residue (Corresponding to the Ile54 site of GmGSTU10) is substituted by Thr in SbGSTU7 (Figure 6B). In order to verify whether this substitution was responsible for the low enzymatic activities of the SbGSTU7 protein, two groups of mutants were constructed. Firstly, the Thr53 residue of SbGSTU7 was, respectively, mutated to Ile and Val residues. Compared with SbGSTU7, the enzymatic activities of the mutants T53V and T53I to the CDNB, NBD$\mathrm{Cl}$ and fluorodifen substrates were much higher (Figure 7A). Secondly, this study mutated the Ile55 residue of SbGSTU6 to a Thr residue. Compared with SbGSTU6, the mutant I55T showed much lower enzymatic activities to the CDNB, NBD-Cl and fluorodifen substrates (Figure 7B). 

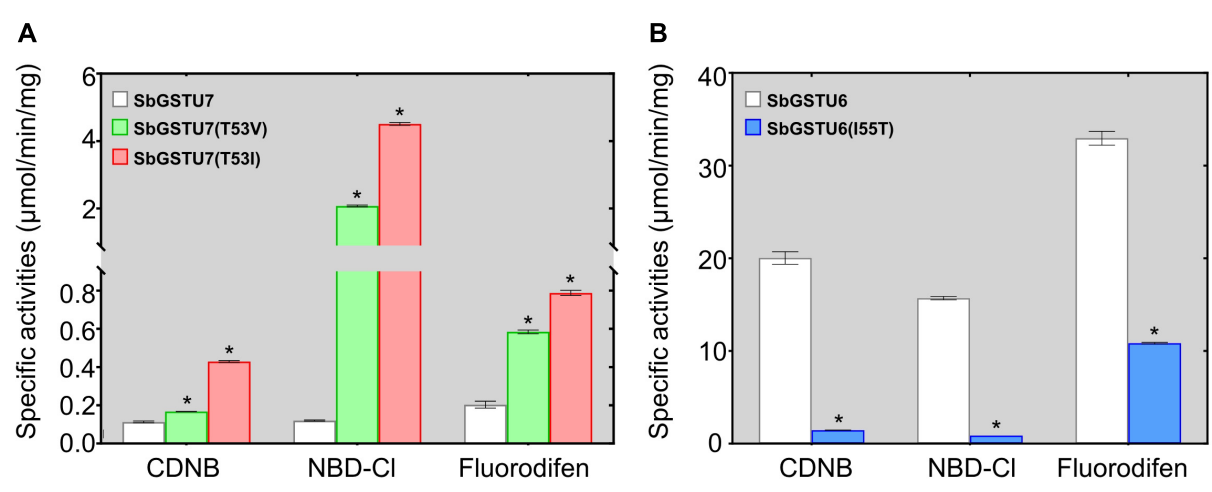

FIGURE 7 | Enzymatic activities of the SbGSTU7 mutants (A) and SbGSTU6 mutant (B). Data for each sample represent the average of three biological replicates, and bars indicate the standard deviation; ${ }^{*}$ significant difference (one tail $t$-test, $P<0.01$ ) in enzymatic activities between the wild-type and its mutants.

\section{DISCUSSION}

Twenty-two full-length GST genes were cloned from Salix babylonica genome. These 22 Salix GSTs were divided into tau, phi and DHAR classes. The previous study identified 81 GST genes from the Populus trichocarpa genome (Lan et al., 2009), which were divided into eight classes, including the theta, zeta, phi, tau, lambda, DHAR, TCQD, and EF1B $\gamma$ GST classes. Compared with the other class GSTs, the tau GST class contained the highest number of members. Similar to Populus trichocarpa, the Salix tau GSTs contained the highest number of members among these Salix GSTs. The gene structures of the tau and phi GSTs in Salix were conserved with their homologs in Populus (Lan et al., 2009), and their enzymatic characteristics were also similar to those of Populus. For example, the Salix tau and phi GSTs had a broad substrate spectrum and differentiated substrate activities. In addition, both Salix and Populus DHAR showed high activity toward the DHA substrate, which distinctly diverged in the enzyme specificity from the other GST classes.

Regarding the GST proteins, there are two distinct domains: The N-terminal and the C-terminal. The N-terminal domain contains $\alpha$-helices and $\beta$-strands, which are arranged in a thioredoxin-like fold. The C-terminal domain is all $\alpha$-helical. The two domains are connected by a short linker sequence. This study confirmed that the sequences similarities between the N-terminal domain sequences of Salix tau and phi GSTs were higher than those of the C-terminal domain sequences (Figure 3), which meant that the N-terminal domain sequences were much more conserved. Similar results were previously observed in other plant GSTs (Lan et al., 2009; Yang et al., 2014; Liu et al., 2015). GSTs catalyze the conjugation of the glutathione thiolate anion with a multitude of second substrates or as non-covalent binding proteins for a range of hydrophobic ligands (Frova, 2003). Besides, the N-terminal domain of GSTs contains a glutathione binding site (G-site) for the common GST substrate (Dirr et al., 1994). These characteristics resulted in highly conserved $\mathrm{N}$-terminal domain structures among different GSTs. On the other hand, the C-terminal domain contains a hydrophobic substrate binding site (H-site) (Dirr et al., 1994), and it could accommodate a diverse range of hydrophobic compounds (Edwards et al., 2000). The diversity of substrates correlates to variable C-terminal domain structures.

In this study, we determined the enzymatic activity of the Salix GST proteins. Interestingly, only theGSTU13 protein of Salix showed GSH peroxidase activity whereas many GSTs of other species usually presented this activity (Liu et al., 2015; Han et al., 2018). It is possible that the other GSTs of Salix could also have GSH peroxidase activity but they are too low to be detected under our measurement conditions or they have GSH peroxidase activity toward different substrates other than cumene hydroperoxide but are not detected in this study. Among the 14 Salix tau GSTs, SbGSTU7 showed the lowest enzymatic activities to the CDNB, NBD-Cl and fluorodifen substrates. The Thr53 of SbGSTU7 was a G-site residue, while this residue was Ile/Val in all the other Salix tau GSTs. Compared with SbGSTU7, the enzymatic activities of the T53V and T53I mutants to the CDNB, NBD-Cl and fluorodifen substrate were much higher (Figure 7A). These results indicated that the Ile/Val $\rightarrow$ Thr substitution could result in the low enzymatic activities of SbGSTU7. Thr53 is located in the loop that connects the $\alpha$-helix 2 to the $\beta$-strand 3 in the $\mathrm{N}$-terminal domain (Figure 6A) and plays important roles in the recognition and orientation of GSH (Dirr et al., 1994). Pro54, a neighboring residue of Thr53, is important for the proper folding and packing of the G-site substructures (Zeng et al., 2005), and it was highly conserved in all the Salix tau GSTs (Figure 6B). The Ile/Val $\rightarrow$ Thr substitution might alter the conformation of Pro54 and the loop structure connecting the $\alpha$-helix 2 to the $\beta$-strand 3 , and these changes might affect the conformation of the G site in SbGSTU7.

\section{CONCLUSION}

Twenty-two full-length GST genes were cloned from Salix babylonica genome. The Salix tau and phi GST proteins showed substantially different tissue-specific expression patterns. In addition, the GST proteins exhibited significant variations in the enzymatic activity. The Ile/Val $\rightarrow$ Thr substitution resulted in the lowest activity of SbGSTU7 among the Salix GSTs. This study suggest that an amino acid at the putative glutathione-binding 
site may play an important role in the divergence of enzymatic functions of Salix GST family.

\section{DATA AVAILABILITY STATEMENT}

The sequence data of GSTs identified in this study was deposited in the National Center for Biotechnology Information (NCBI) under the accession numbers listed in Table $\mathbf{1}$.

\section{AUTHOR CONTRIBUTIONS}

H-LY and X-LZ conceived the project. X-LZ and Z-JX performed research. All authors contributed to data analysis, writing of the manuscript, and reviewed the manuscript.

\section{REFERENCES}

Allocati, N., Federici, L., Masulli, M., and Di Ilio, C. (2009). Glutathione transferases in bacteria. FEBS J. 276, 58-75. doi: 10.1111/j.1742-4658.2008. 06743.x

Alzohairy, A. (2011). BioEdit: an important software for molecular biology. GERF Bull. Biosci. 2, 60-61. doi: 10.1016/j.compbiolchem.2019.02.002

Armstrong, R. N. (1997). Structure, catalytic mechanism, and evolution of the glutathione transferases. Chem. Res. Toxicol. 10, 2-18. doi: 10.1021/tx960072x

Basantani, M., and Srivastava, A. (2007). Plant glutathione transferases - a decade falls short. Can. J. Bot. 85, 443-456. doi: 10.1139/B07-033

Benekos, K., Kissoudis, C., Nianiou-Obeidat, I., Labrou, N., Madesis, P., Kalamaki, M., et al. (2010). Overexpression of a specific soybean GmGSTU4 isoenzyme improves diphenyl ether and chloroacetanilide herbicide tolerance of transgenic tobacco plants. J. Biotechnol. 150, 195-201. doi: 10.1016/j.jbiotec.2010.07.011

Brereton, N. J., Ray, M. J., Shield, I., Martin, P., Karp, A., and Murphy, R. J. (2012). Reaction wood - a key cause of variation in cell wall recalcitrance in willow. Biotechnol. Biofuels 5:83. doi: 10.1186/1754-6834-5-83

Cummins, I., Dixon, D. P., Freitag-Pohl, S., Skipsey, M., and Edwards, R. (2011). Multiple roles for plant glutathione transferases in xenobiotic detoxification. Drug Metab. Rev. 43, 266-280. doi: 10.3109/03602532.2011.552910

Cummins, I., Wortley, D. J., Sabbadin, F., He, Z., Coxon, C. R., Straker, H. E., et al. (2013). Key role for a glutathione transferase in multiple-herbicide resistance in grass weeds. Proc. Natl. Acad. Sci. U.S.A. 110, 5812-5817. doi: 10.1073/pnas. 1221179110

Cunniff, J., Purdy, S. J., Barraclough, T. J., Castle, M., Maddison, A. L., Jones, L. E., et al. (2015). High yielding biomass genotypes of willow (Salix spp.) show differences in below ground biomass allocation. Biomass Bioenergy 80, 114-127. doi: 10.1016/j.biombioe.2015.04.020

Dickmann, D. I, and Kuzovkina, J. (2014). "Poplars and willows of the world, with emphasis on silviculturally important species," in Poplars and Willows: Trees for Society and the Environment, eds J. G. Isebrands and J. Richardson (London: CAB International and FAO), 8-83.

Dirr, H., Reinemer, P., and Huber, R. (1994). X-ray crystal structures of cytosolic glutathione S-transferases. Implications for protein architecture, substrate recognition and catalytic function. Eur. J. Biochem. 220, 645-661. doi: 10.1111/ j.1432-1033.1994.tb18666.x

Dixon, D. P., Skipsey, M., and Edwards, R. (2010). Roles for glutathione transferases in plant secondary metabolism. Phytochemistry 71, 338-350. doi: 10.1016/j.phytochem.2009.12.012

Edwards, R., and Dixon, D. P. (2005). Plant glutathione transferases. Methods Enzymol. 401, 169-186. doi: 10.1016/S0076-6879(05)01011-6

Edwards, R., Dixon, D. P., and Walbot, V. (2000). Plant glutathione S-transferases: enzymes with multiple functions in sickness and in health. Trends Plant Sci. 5, 193-198. doi: 10.1016/S1360-1385(00)01601-0

\section{FUNDING}

This study was supported by grants from the National Natural Science Foundation of China (NSFC 31570664).

\section{SUPPLEMENTARY MATERIAL}

The Supplementary Material for this article can be found online at: https://www.frontiersin.org/articles/10.3389/fpls.2020.00364/ full\#supplementary-material

TABLE S1 | Primers used to amplify Salix babylonica GST genes.

TABLE S2 | PCR primers used to detect the expression of Salix babylonica GST genes.

TABLE S3 | Primers used to construct the protein expression vector.

Frova, C. (2003). The plant glutathione transferase gene family: genomic structure, functions, expression and evolution. Physiologia Plant. 119, 469-479. doi: 10. 1046/j.1399-3054.2003.00183.x

Frova, C. (2006). Glutathione transferases in the genomics era: new insights and perspectives. Biomol. Eng. 23, 149-169. doi: 10.1016/j.bioeng.2006.05.020

Guindon, S., Delsuc, F., Dufayard, J. F., and Gascuel, O. (2009). Estimating maximum likelihood phylogenies with PhyML. Methods Mol. Biol. 537, 113137. doi: 10.1007/978-1-59745-251-9_6

Habig, W. H., Pabst, M. J., and Jakoby, W. B. (1974). Glutathione S-transferases. The first enzymatic step in mercapturic acid formation. J. Biol. Chem. 249, 7130-7139.

Han, X. M., Yang, Z. L., Liu, Y. J., Yang, H. L., and Zeng, Q. Y. (2018). Genome-wide profiling of expression and biochemical functions of the Medicago glutathione S-transferase gene family. Plant Physiol. Biochem. 126, 126-133. doi: 10.1016/j. plaphy.2018.03.004

Jiang, H. W., Liu, M. J., Chen, I. C., Huang, C. H., Chao, L. Y., and Hsieh, H. L. (2010). A glutathione S-transferase regulated by light and hormones participates in the modulation of Arabidopsis seedling development. Plant Physiol. 154, 1646-1658. doi: 10.1104/pp.110.159152

Labrou, N. E., Papageorgiou, A. C., Pavli, O., and Flemetakis, E. (2015). Plant GSTome: structure and functional role in xenome network and plant stress response. Curr. Opin. Biotechnol. 32, 186-194. doi: 10.1016/j.copbio.2014.12. 024

Lan, T., Yang, Z. L., Yang, X., Liu, Y. J., Wang, X. R., and Zeng, Q. Y. (2009). Extensive functional diversification of the Populus glutathione S-transferase supergene family. Plant Cell 21, 3749-3766. doi: 10.1105/tpc.109.070219

Liu, H. J., Tang, Z. X., Han, X. M., Yang, Z. L., Zhang, F. M., Yang, H. L., et al. (2015). Divergence in enzymatic activities in the soybean GST supergene family provides new insight into the evolutionary dynamics of whole-genome duplicates. Mol. Biol. Evol. 32, 2844-2859. doi: 10.1093/molbev/msv156

Liu, Y. J., Han, X. M., Ren, L. L., Yang, H. L., and Zeng, Q. Y. (2013). Functional divergence of the glutathione S-transferase supergene family in Physcomitrella patens reveals complex patterns of large gene family evolution in land plants. Plant Physiol. 161, 773-786. doi: 10.1104/pp.112.205815

Livak, K. J., and Schmittgen, T. D. (2001). Analysis of relative gene expression data using real-time quantitative PCR and the 2(-Delta Delta C(T)) Method. Methods 25, 402-408. doi: 10.1006/meth.2001.1262

Loyall, L., Uchida, K., Braun, S., Furuya, M., and Frohnmeyer, H. (2000). Glutathione and a UV light-induced glutathione S-transferase are involved in signaling to chalcone synthase in cell cultures. Plant Cell 12, 1939-1950. doi: $10.1105 /$ tpc. 12.10 .1939

Oakley, A. J. (2005). Glutathione transferases: new functions. Curr. Opin. Struct. Biol. 15, 716-723. doi: 10.1016/j.sbi.2005.10.005

Ricci, G., Caccuri, A. M., Lo Bello, M., Pastore, A., Piemonte, F., and Federici, G. (1994). Colorimetric and fluorometric assays of glutathione transferase based 
on 7-chloro-4-nitrobenzo-2-oxa-1,3-diazole. Anal. Biochem. 218, 463-465. doi: 10.1006/abio.1994.1209

Roxas, V. P., Lodhi, S. A., Garrett, D. K., Mahan, J. R., and Allen, R. D. (2000). Stress tolerance in transgenic tobacco seedlings that overexpress glutathione S-transferase/glutathione peroxidase. Plant Cell Physiol. 41, 1229-1234. doi: $10.1093 / \mathrm{pcp} / \mathrm{pcd} 051$

Skipsey, M., Cummins, I., Andrews, C. J., Jepson, I., and Edwards, R. (2005). Manipulation of plant tolerance to herbicides through co-ordinated metabolic engineering of a detoxifying glutathione transferase and thiol cosubstrate. Plant Biotech. J. 3, 409-420. doi: 10.1111/j.1467-7652.2005.00134.x

Wu, J., Nyman, T., Wang, D. C., Argus, G. W., Yang, Y. P., and Chen, J. H. (2015). Phylogeny of Salix subgenus Salix s.l. (Salicaceae): delimitation, biogeography, and reticulate evolution. BMC Evol. Biol. 15:31. doi: 10.1186/s12862-015-03117

Yang, Q., Liu, Y. J., and Zeng, Q. Y. (2014). Biochemical functions of the glutathione transferase supergene family of Larix kaempferi. Plant Physiol. Biochem. 77, 99-107. doi: 10.1016/j.plaphy.2014.02.003

Yildirim, K., and Kasim, G. C. (2016). Phytoremediation potential of poplar and willow species in small scale constructed wetland for boron removal. Chemosphere 194, 722-736. doi: 10.1016/j.chemosphere.2017 12.036

Zeng, Q. Y., Lu, H., and Wang, X. R. (2005). Molecular characterization of a glutathione transferase from Pinus tabulaeformis (Pinaceae). Biochimie 87, 445-455. doi: 10.1016/j.biochi.2005.01.002

Zeng, Q. Y., and Wang, X. R. (2005). Catalytic properties of glutathionebinding residues in a tau class glutathione transferase (PtGSTU1) from Pinus tabulaeformis. FEBS Lett. 579, 2657-2662. doi: 10.1016/j.febslet.2005.03.086

Conflict of Interest: The authors declare that the research was conducted in the absence of any commercial or financial relationships that could be construed as a potential conflict of interest.

Copyright (c) 2020 Zhuge, Xu, Xiu and Yang. This is an open-access article distributed under the terms of the Creative Commons Attribution License (CC BY). The use, distribution or reproduction in other forums is permitted, provided the original author(s) and the copyright owner(s) are credited and that the original publication in this journal is cited, in accordance with accepted academic practice. No use, distribution or reproduction is permitted which does not comply with these terms. 